\title{
Bright-exciton splittings in inorganic cesium lead halide perovskite nanocrystals
}

\author{
R. Ben Aich ${ }^{1}$ I. Saïdi ${ }^{1}$, S. Ben Radhia ${ }^{1}$, K. Boujdaria ${ }^{1}{ }^{*}$ T. Barisien ${ }^{2}$, \\ L. Legrand ${ }^{2}$, F. Bernardot ${ }^{2}$, M. Chamarro ${ }^{2}$, and C. Testelin ${ }^{2}$ \\ ${ }^{1}$ Université de Carthage, Faculté des Sciences de Bizerte, LR01ES15, \\ Laboratoire de Physique des Matériaux : Structure et Propriétés, \\ 7021 Zarzouna, Bizerte, Tunisia and \\ ${ }^{2}$ Sorbonne Université, CNRS-UMR 7588, \\ Institut des NanoSciences de Paris, INSP, \\ 4 place Jussieu, F-75005, Paris, France
}

(Dated: February 20, 2019)

\begin{abstract}
Since their first synthesis in 2015, the all-inorganic lead halide perovskite nanocrystals $\mathrm{CsPbX}_{3}$ $(X=C l, B r, I)$ have attracted a great attention due to their outstanding electronic and optical properties as well as their performances which outclass the ones of II-VI conterparts in many application fields. In addition to these properties, the understanding of the emission features in these systems at the single object scale is crucial e.g. for nanophotonics and quantum optics devices. The details of the band-edge excitonic emission are here theoretically explored. The contribution of the long-range exchange interaction to the bright-exciton splittings is computed in strong and weak confinement regimes by using the group theory and k.p arguments. We show that the shape anisotropy of a nanocrystal can also be at play with the crystalline (cubic, tetragonal or orthorhombic) structures to explain the emission properties. In the weak confinement regime, splittings are inversely proportional to the cube of the exciton Bohr radius and we observe an increase of the splittings from iodide, to bromide, then chloride perovskite compounds. However, in the strong confinement regime, splittings increase inversely proportional to the nanocrystal volume and, for a given nanocrystal size, the splitting values are comparable for the three halide perovskite materials. The present theoretical developments lead to quantitative contributions in good agreement with available experimental data mainly in the weak confinement regime.
\end{abstract}

PACS numbers:

*Electronic address: kais.boujdaria@fsb.rnu.tn 


\section{INTRODUCTION}

Cesium lead halide perovskite nanocrystals (NCs) have recently emerged as an interesting alternative to II-VI material-based NCs, which have monopolized the attention of researchers over the last twenty years. Thanks to defect-tolerance behaviour [1], the halide perovskite NCs present a high luminescence yield (50\% -90\% at room temperature) without the constraint to synthesize "core-shell" structures $[2,3]$. Their inorganic structure $\left(\mathrm{CsPBX}_{3}\right.$ where $\mathrm{X}$ is a halide anion $\left(\mathrm{Cl}^{-}, \mathrm{Br}^{-}, \mathrm{I}^{-}\right)$) also makes them relatively stable systems compared to organic-inorganic hybrid perovskites. They have unique optical properties: very bright emission with an energy easily tunable from the ultraviolet to the infrared range by combining substitution of halogen atoms and quantum confinement. Since their first synthesis, in 2015 [4], we assist to a skyrocketing scientific interest in these NCs. Recently, taking advantage of all these properties, various studies have explored perovskite NC applications in low-threshold lasers [5-7], LED emitters [8], photodetectors [9] and single photon emission $[9-12]$.

An exhaustive understanding of the band-edge exciton states is fundamental to explain the excellent optical properties of these NCs and to investigate their high potential to work in nanophotonics and quantum optics or quantum information devices. Indeed, several proposed quantum devices are very much concerned with the existence of the brightexciton splittings. For example, in propositions exploiting the two distinguishable paths in the biexciton-exciton transition, this splitting becomes detrimental for the generation of polarization-entangled photon-pair if the splitting is larger than the linewidth of emitting states [13-16]. However, it is also possible to benefit from the bright-exciton splitting to build a two-bit conditional quantum logic gate in the two excitons configuration [17].

First experimental studies of low temperature photoluminescence (PL) on single halide perovskite $\mathrm{NC}$ without confinement effects have evidenced a fine structure of bright excitons: narrow lines grouped in a triplet [9]. More recent studies have shown the presence of triplets [18-21], doublets $[18,19,21,22]$ or single lines [21]. However, very few theoretical studies have been done $[18,19,21]$. In particular, Becker et al [21] explain explain these experimental results by the presence of a Rashba effect related to inversion symmetry breaking on a local scale resulting from dynamical fluctuations of $\mathrm{Cs}^{+}$ions positions in the inorganic perovskite lattice. In contrast to authors of references $[18,19]$ they do not consider supplementary 
breaking of symmetry.

The bulk band structure of $\mathrm{CsPbX}_{3}$ and hybrid perovskites has been theoretically addressed in the past [23] and recently [4, 21, 24-26]. They are direct band-gap semiconductors with a reverse ordering of band-edge states as compared with more conventional semiconductors. The band-edge excitons are Coulomb bound states, pairing a hole in the upper valence band (VB) $\left(j_{h}=1 / 2, j_{z}^{h}= \pm 1 / 2\right)$ and an electron from the lowest split-off conduction band (CB) $\left(j_{e}=1 / 2, j_{z}^{e}= \pm 1 / 2\right)$ leading to four exciton states. The exciton states are split into several energy sublevels, named the exciton fine structure, as a result of the electron-hole exchange interaction (e-h EI) which is basicaly related to the Coulomb interaction. The exchange energy depends on the distance between the electrons and holes or more precisely, on the overlap of their wave functions. In semiconductor NCs, e-h EI, as well as direct Coulomb interaction, are greatly enhanced by quantum confinement due to the increased spatial overlap of the electron and hole wave functions [27-31]. In the framework of the effective mass approximation, the theory of the excitonic fine structure splittings due to the e-h EI in bulk semiconductor was put forward by Pikus and Bir [32, 33] and Denisov and Makarov [34]. Two main contributions can be considered, namely the short-range (SR) part and the long-range (LR) part as defined and discussed in Refs. [32, 33], after separation in the momentum space. Note that these SR and LR contributions are also named analytical and non-analytical contributions [34]. As discussed in Refs [35-37], the LR (non-analytical) part contains a contact term, sometimes included in the SR contribution. The theoretical reexamination of the e-h EI in NCs of « classical » semiconductors has been carried out $[35,38-42]$ in different works and, in particular, a special attention has been carried out to elucidate the LR and SR contributions to the exciton fine structure [43-46].

The $\mathrm{CsPbX}_{3}$ perovskite NCs associate a conduction- and valence-bands ordering reversed with respect to more regular semiconductors and the presence of different crystallographic phases [18, 47-51]: the high temperature cubic phase $\left(O_{h}\right.$ point group), the tetragonal phase ( $D_{4 h}$ point group), and the low-temperature orthorhombic phase $\left(D_{2 h}\right.$ point group). Even another low-temperature monoclinic phase has been also observed [51] by neutron scattering for $\mathrm{CsPbCl}_{3}$. In inorganic bulk perovskites, all phase transitions occur well above room temperature $[50,51]$. An elaborate review of the perovskites symmetry can be found in the literature $[52,53]$. However, a detailed knowledge of the point-group symmetry and an appropriate irreductible representations become crucial to describe the band-edge excitons 
of highly anisotropic perovskite NCs.

For an isotropic NC with a cubic crystal phase, the fourfold degenerate excitons states constituted by the hole states of the upper VB and the electron states of the lower CB are splitted by e-h EI leading to an optically forbidden singlet state and three degenerate optically allowed exciton states. Becker et al [21] have calculated that the SR contribution to the bright-dark exciton splitting is of the order of hundreds of $\mu \mathrm{eV}$. Very recently, Nestoklon et al [54] have calculated the LR contribution to bright exciton splitting of shape-anisotropic halide perovskite NCs with $O_{h}$ as the point group. These authors have shown that an anisotropic shape is at the origin of the appearence of a doublet of bright exciton states in good agreement with experimental results of exciton optical orientation and alignement. However, for perfectly cubic shape NCs with $O_{h}$ point symmetry, there are no bright exciton splittings.

In this work, we have taken into account actual crystal structures of perovskites NCs in order to reach a fine description of this new class of materials and provide valuable informations for future applications in quantum nanodevices. We calculated the LR part of eh EI and its contribution to the bright exciton splittings for anisotropic halide perovskite NCs in the strong and in the weak confinement regimes. Two different origins of the reduction of symmetry are considered: (i) the anisotropy of the NC shape (as in Ref. [54]), (ii) the anisotropy of the crystalline phase of NCs. We do not address the SR contribution to the bright exciton splitting. In fact, for cubic-shaped NCs the SR interaction may have a significant contribution only for orthorhombic phase $\left(D_{2 h}\right.$ point group) [54]. We show that doublets or triplets in the PL of a single NC can be observed even in a symmetric cristal phase like $O_{h}$ due to anisotropic shapes. When isotropic shapes are considered, a cubic phase $\left(O_{h}\right.$ point group) leads to three degenerate bright exciton states, a tetragonal phase $\left(D_{4 h}\right.$ point group) to a doublet and orthorhombic phase $\left(D_{2 h}\right.$ point group) to a non-degenerate triplet exciton state. In a more general situation, bright-exciton fine structure is the result of an interplay of the shape and crystalline phase anisotropies. We draw an exhaustive panorama of the bright-exciton fine structure of all the more studied inorganic perovksite NCs which constitutes a valuable information to stimulate prospects on optimal quantum device performances based on these materials. 


\section{THEORETICAL MODEL}

The expression of the LR e-h Hamiltonian is written as follows [46]:

$\underset{\substack{m^{\prime} n^{\prime} \\ m n}}{L R}\left(\begin{array}{ll}r_{e}^{\prime} & r_{h}^{\prime} \\ r_{e} & r_{h}\end{array}\right)=\frac{1}{(2 \pi)^{3}} \int d \mathbf{q}\left(\frac{e^{2}}{\epsilon_{0} \epsilon_{X}} \frac{1}{q^{2}}\right)\left(\sum_{i, j} \underset{\substack{\mathcal{K}_{n^{\prime}} \mathcal{K}_{m} \\ \mathcal{M}^{\prime}}}{i j} q_{i} q_{j}\right) \exp \left[i \mathbf{q} \cdot\left(\mathbf{r}_{e}-\mathbf{r}_{e}^{\prime}\right)\right] \delta\left(\mathbf{r}_{e}-\mathbf{r}_{h}\right) \delta\left(\mathbf{r}_{e}^{\prime}-\mathbf{r}_{h}^{\prime}\right)$

in which $m, m^{\prime}\left(n, n^{\prime}\right)$ label the Bloch states of the electron in the CB (the hole in the VB), $\left(\mathbf{r}_{e}, \mathbf{r}_{e}^{\prime}\right)$ and $\left(\mathbf{r}_{h}, \mathbf{r}_{h}^{\prime}\right)$ denote the coordinates of the electrons and holes, respectively. $\epsilon_{X}$ is the high-frequency (at the exciton frequency) dielectric constant. Including the spin degree of

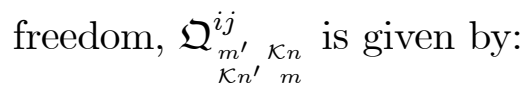

$$
\mathfrak{Q}_{\substack{\mathcal{K}^{\prime}{ }^{\prime} \mathcal{K}_{n} \\ i j}}^{i j}=\frac{\hbar^{2}}{m_{0}^{2}} \frac{\left\langle m^{\prime}\left|p_{i}\right| \mathcal{K} n^{\prime}\right\rangle\left\langle\mathcal{K} n\left|p_{j}\right| m\right\rangle}{\left(E_{m}^{0}-E_{n}^{0}\right)\left(E_{m^{\prime}}^{0}-E_{n^{\prime}}^{0}\right)}
$$

with $p_{i}\left(p_{j}\right)$ is the $i(j)$ component of the $\mathbf{p}$ momentum, $m_{0}$ is the free electron mass, $E_{\nu}^{0}$ $\left(\nu=m, m^{\prime}, n, n^{\prime}\right)$ is the energy of the $\nu t h$ band, and $\mathcal{K}$ is the time-reversal operator. Note that $\mathcal{K}$ leaves $\mathbf{r}$ unchanged, changes the momentum $\mathbf{p}$ to $(-\mathbf{p})$, and changes the angular momentum to their opposite, in particular it changes Pauli operators $\sigma$ to $(-\sigma)$. For each point group considered in this work, the explicit form of the matrix representation $\mathfrak{Q}_{\substack{\mathcal{K}^{\prime}{ }^{\prime} \mathcal{K}_{n} \\ m}}(\mathbf{q})=\sum_{i, j} \mathfrak{Q}_{\substack{m_{n^{\prime}} \mathcal{K}_{n} \\ \mathcal{K}_{m}}}^{i j} q_{i} q_{j}$ is given in Appendix B.

The exciton wave functions in NCs are the product of the Bloch functions and the exciton envelope wave function $\Psi_{X}\left(\mathbf{r}_{e}, \mathbf{r}_{h}\right)$, which describes the spatial motion of the exciton confined in the NC. In our modelization, we have taken into account the upper VB $\left(j_{h}=1 / 2, j_{z}^{h}= \pm 1 / 2\right)$ and the lowest split-off $\mathrm{CB}\left(j_{e}=1 / 2, j_{z}^{e}= \pm 1 / 2\right)$. We denote the hole Bloch wave functions as $\left|W_{1}\right\rangle=|1 / 2,1 / 2\rangle,\left|W_{2}\right\rangle=|1 / 2,-1 / 2\rangle$, and the CB Bloch wave functions as $\left|C_{1}\right\rangle=|1 / 2,1 / 2\rangle,\left|C_{2}\right\rangle=|1 / 2,-1 / 2\rangle$. The basis Bloch wave functions for conduction- and valence-bands are given explicitly in Appendix A. In what follows, we will model the envelope wave function of a particle (electron, hole or exciton) confined in the rectangular parallelepiped-shape $\mathrm{NC}$ by:

$$
\Psi\left(\mathbf{r}_{e, h}\right)=\sqrt{\frac{2^{3}}{L_{x} L_{y} L_{z}}} \cos \left(\pi x_{e, h} / L_{x}\right) \cos \left(\pi y_{e, h} / L_{y}\right) \cos \left(\pi z_{e, h} / L_{z}\right)
$$

where $L_{x}, L_{y}$ and $L_{z}$ are the edge lenghts of the rectangular parallelepiped-shape NC. 
One distinguishes here two distinct regimes of exciton confinement in a NC. In the strongconfinement (sc) regime, when the size of the NC is less than the exciton Bohr diameter, we take $\Psi_{X}^{s c}\left(\mathbf{r}_{e}, \mathbf{r}_{h}\right)=\Psi\left(\mathbf{r}_{e}\right) \Psi\left(\mathbf{r}_{h}\right)$, where $\Psi\left(\mathbf{r}_{e}\right)$ and $\Psi\left(\mathbf{r}_{h}\right)$ are given by Eq. (3). In the weak-confinement (wc) regime, when the NC size is large compared with the exciton Bohr radius $\left(a_{X}\right)$, the exciton is localized within the $\mathrm{NC}$ as a whole and we take:

$$
\Psi_{X}^{w c}\left(\mathbf{r}_{e}, \mathbf{r}_{h}\right)=\Psi(\mathbf{R}) \Phi\left(\mathbf{r}_{e}-\mathbf{r}_{h}\right)
$$

in which $\mathbf{R}$ is the exciton mass-center, and $\Phi$ the hydrogenoid function, with $\Phi(0)=\frac{1}{\sqrt{\pi a_{X}^{3}}}$. In the bulk (b) regime, the exciton wave function is written :

$$
\Psi_{X}^{b}\left(\mathbf{r}_{e}, \mathbf{r}_{h}\right)=\frac{1}{\sqrt{V}} \exp (i \mathbf{K} . \mathbf{R}) \Phi\left(\mathbf{r}_{e}-\mathbf{r}_{h}\right)
$$

with $\mathbf{K}$ the exciton momentum ( $\mathbf{K}=\mathbf{0}$ in the ground state). Note that, for the bulk, the e-h exchange coupling will be equal to the one obtained in the weak confinement regime, for an isotropic $\mathrm{NC}\left(L_{x}=L_{y}=L_{z}\right)$.

As quoted in Ref. [54], "In a NC of highly anisotropic shape, even when the crystal structure is cubic, the symmetry allows for the full splitting of the bright excitonic level into the $|X\rangle,|Y\rangle$, and $|Z\rangle$ states". Then, we consider a symmetry reduction due to NC shape anisotropy or/and, going further, a crystal symmetry lowering from $O_{h}$ to $D_{4 h}$ or $D_{2 h}$. In those cases, the fine structure of the bright-exciton can be found analytically from the $\mathcal{H}_{\text {exch }}^{L R}$ matrix.

In the sub-space of bright triplet states $\left(j=j_{e}+j_{h}=1\right)$, the basis elements are $\left\{|+1\rangle,|-1\rangle,\left|O_{B}\right\rangle\right\}$. The bright triplet states and the dark singlet state $(j=0),\left|O_{D}\right\rangle$, are given explicitly in Appendix B. Substituting the square of the Fourier transform of the exciton wave function $\left(\Psi_{X}^{\alpha}\left(\mathbf{r}_{e}=\mathbf{R}, \mathbf{r}_{h}=\mathbf{R}\right), \alpha=s c, w c, b\right)$ into Eq. (1), the LR e-h EI matrix given in Eq. (B2) of appendix B is reduced, in the bright-state basis $\left\{|+1\rangle,|-1\rangle,\left|O_{B}\right\rangle\right\}$, to:

$$
\mathcal{H}_{\text {exch }}^{L R}=\left[\begin{array}{ccc}
\Sigma_{d} & \Sigma_{o d} & 0 \\
\Sigma_{o d} & \Sigma_{d} & 0 \\
0 & 0 & \Sigma_{z}
\end{array}\right]
$$

The detailed derivation of $\mathcal{H}_{\text {exch }}^{L R}$ matrix for each point group is given in Appendix B. It is then straightforward to calculate the fine anisotropic splitting in diagonalizing the LR 
exchange matrix given by Eq. (6). It is clear that, for a general reduction of the symmetry case, all excitonic levels are non-degenerate and the bright excitonic levels are split into three non-degenerate states. The exciton energy labeling of the fine structure states is given in Fig. 1a. Fig. 1b shows the NC shape and the parameters $L_{x}, L_{y}, L_{z}, r$ and $s$ used in our calculations.

The bright-states energies are expressed in terms of the $\Sigma_{d}, \Sigma_{o d}$ and $\Sigma_{z}$ energies and the eigenenergies of $\mathcal{H}_{\text {exch }}^{L R}$ are $\Sigma_{1}=\left(\Sigma_{d}-\Sigma_{o d}\right), \Sigma_{2}=\left(\Sigma_{d}+\Sigma_{o d}\right)$, and $\Sigma_{3}=\Sigma_{z}$. In appendix B, we also give explicitly the different expressions of $\Sigma_{\ell}(\ell=d$, od, $z) . \Sigma_{d}, \Sigma_{o d}$ and $\Sigma_{z}$ depend at first on the intrinsic properties of the NC, namely the point group of the $\mathrm{NC}$ constituent materials, and secondly on the extrinsic properties of the NC, namely their anisotropic shape. When all the bright excitonic energy levels $\Sigma_{1}, \Sigma_{2}$, and $\Sigma_{3}$ are distinct, they can be characterized by the two values of anisotropic splittings. In what follows, we denoted the smallest and the largest of these values by $\Delta E_{1}=\left(\Sigma_{2}-\Sigma_{3}\right)$ and $\Delta E_{2}=\left(\Sigma_{1}-\Sigma_{3}\right)$ (see Fig. $1 \mathrm{a})$.
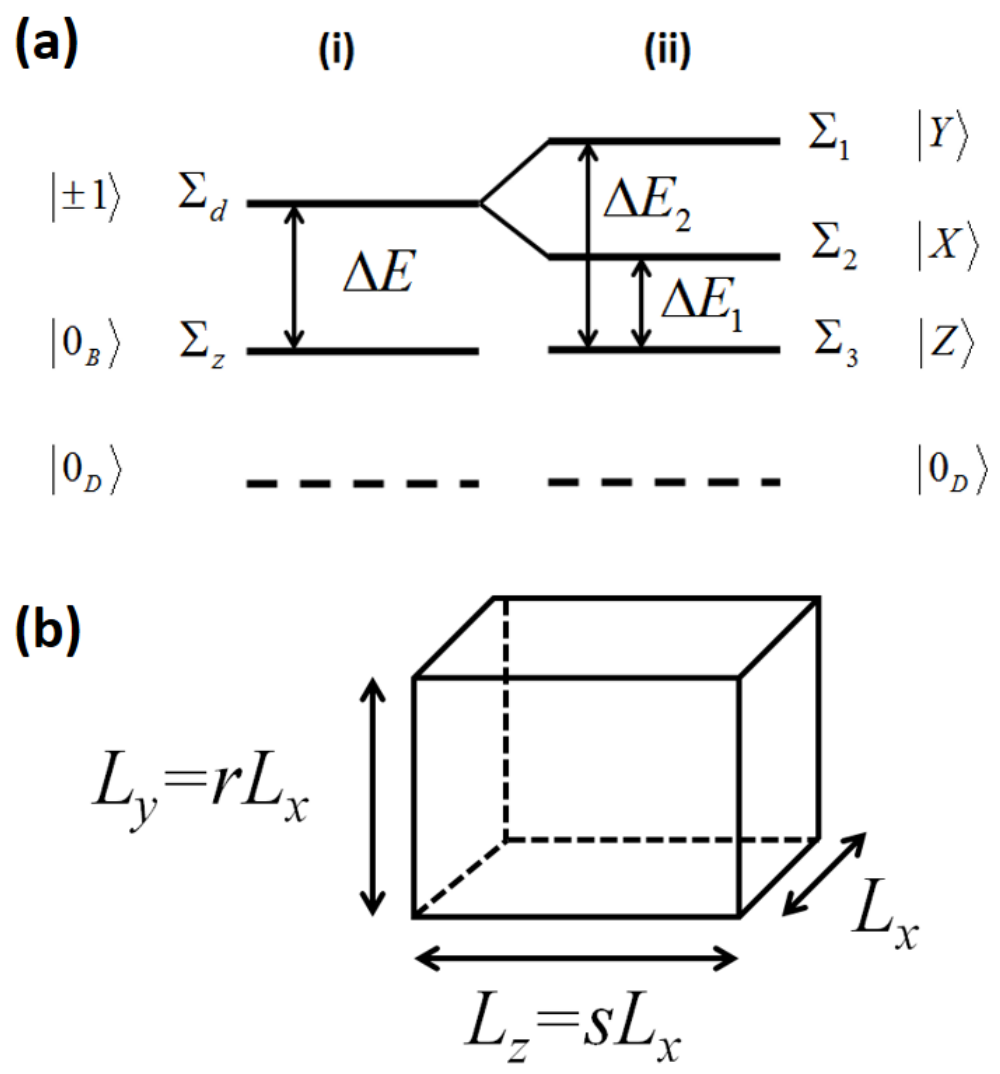

Fig. 1: (a) Energy labeling of the fine structure states. For the parameters used in this work : i) case of doublet, ii) case of triplet $|X\rangle,|Y\rangle,|Z\rangle$; (b) The NC shape and their corresponding parameters $\left(L_{x}, L_{y}, L_{z}, r, s\right)$ used in our calculations. 


\begin{tabular}{ccccccccc}
\hline \hline & $E_{g}(\mathrm{eV})$ & $\epsilon_{r}$ & $\mu_{X}\left(m_{0}\right)$ & $a_{X}(\stackrel{\leftrightarrow}{A})$ & $E_{P_{S, \rho}}(\mathrm{eV})$ & $E_{P_{S, z}}(\mathrm{eV})$ & $\epsilon_{X}$ & {$\left[E_{P_{S, \rho}} / E_{g}^{2} \epsilon_{X}\right]\left(\mathrm{eV}^{-1}\right)$} \\
\hline $\mathrm{CsPbI}_{3}{ }^{a}$ & 1.723 & 10 & 0.114 & 46.4 & 23 & 20.7 & $6.32^{d}$ & 1.23 \\
$\mathrm{CsPbBr}_{3}{ }^{a}$ & 2.342 & 7.3 & 0.126 & 30.7 & 28 & 25.2 & $4.96^{d}$ & 1.03 \\
$\mathrm{CsPbCl}_{3}$ & $3.04^{b}$ & 5.03 & $0.125^{c}$ & 21.3 & $30^{c}$ & $27.0^{c}$ & $4.07^{d}$ & 0.80 \\
\hline \hline
\end{tabular}

${ }^{a}$ Reference 56.

${ }^{b}$ Reference 58.

${ }^{c}$ Reference 57.

${ }^{d}$ Reference 4.

TABLE I: Numerical values of the k.p parameters used in this work.

Let us remark that, a fine structure with a dark optical state and two bright components is possible under certain conditions for example in the tetragonal symmetry, in which the k.p parameters are partially anisotropic $\left(P_{S, x}=P_{S, y} \neq P_{S, z}\right.$; see appendix B for the definition of $\left.P_{S, \ell}(\ell=x, y, z)\right)$, assuming an isotropic envelope wave function $\left(L_{x}=L_{y}=L_{z}\right)$. Moreover, a doublet is also possible for the $O_{h}$ point group assuming $L_{y}=L_{z} \neq L_{x}$. We denote the doublet energy by $\Delta E$.

To estimate the values of $\Delta E, \Delta E_{1}$ and $\Delta E_{2}$, we use the bulk parameters of inorganic perovskites summarized in Table I. $E_{g}$ is the bulk band-gap energy. $\epsilon_{r}$ is the effective dielectric constant and $\mu_{X}$ the exciton reduced mass; both are related to the exciton binding energy $E_{X}=\left(\mu_{X} e^{4} / 8 \epsilon_{0}^{2} \epsilon_{r}^{2} h^{2}\right) . a_{X}$ is the exciton Bohr radius defined as $a_{X}=\left(m_{0} / \mu_{X}\right) \epsilon_{r} a_{0}$. $E_{P_{S, \rho}}=\left(2 m_{0} / \hbar^{2}\right) P_{S, \rho}^{2}$ is the related energy to the interband momentum matrix element $\left(P_{S, \rho}\right)$, and can be estimated from the relation $E_{P_{S, \rho}}=(3 / 2)\left(m_{0} / \mu_{X}\right) E_{g}[55]$. In $D_{4 h}$ and $D_{2 h}$ symmetries, we assumed a $10 \%$ anisotropy between the momentum matrix elements and deduced $E_{P_{S, z}}=\left(2 m_{0} / \hbar^{2}\right) P_{S, z}^{2}$. The band parameters at $2 \mathrm{~K}$ of $\mathrm{CsPbI}_{3}$ and $\mathrm{CsPbBr}_{3}$ are extracted from the experimental data given in Table I of Ref. [56]. For $\mathrm{CsPbCl}_{3}$, we have performed a 40-band k.p calculation [57] leading to $E_{P_{S, \rho}}=30 \mathrm{eV}, E_{P_{S, z}}=27 \mathrm{eV}$, and $\mu_{X}=0.125 m_{0}$ (deduced from the couple $\left(m_{e}=0.390 m_{0}, m_{h}=0.184 m_{0}\right)$ ). Taking the measured $E_{g}=3.04 \mathrm{eV}$ [58] and $E_{X}=67 \mathrm{meV}$ [58], we get $\epsilon_{r}=5.03$ and $a_{X}=21.3 \AA$. Finally, $\epsilon_{X}$ is the high-frequency (at exciton resonance) dielectric constant. In the literature, it is very difficult to find an accurate experimental determination of the dielectric constant as a function of the frequency, for inorganic halide perovskites. However, several studies are found for hybrid halide perovskite $[59,60]$ and we observe the following main trends: the $\epsilon_{X}$ value is smaller than the experimentally found $\epsilon_{r}$ [61] and is slightly larger than the calculated high frequency dielectric constant $\epsilon_{\infty}[62]$, while the $\epsilon_{X}$ value decreases with 
increasing $E_{X}$. That is why, we give in Table 1 a lower bound to the $\epsilon_{X}$ by identifying it to the theoretical value of $\epsilon_{\infty}[4]$.

\section{RESULTS}

In the following, we will calculate and discuss the contribution of the LR e-h EI, on the bright exciton splittings, for the three inorganic halide perovskites $\mathrm{CsPbX}_{3}(\mathrm{X}=\mathrm{I}, \mathrm{Br}, \mathrm{Cl})$. From our modelization, we can take into account both intrinsic (or crystallographic) and extrinsic (or shape) anisotropies. We have particularly considered the three different phases: cubic $\left(O_{h}\right)$, tetragonal $\left(D_{4 h}\right)$ and orthorhombic $\left(D_{2 h}\right)$.

In the cubic symmetry $\left(O_{h}\right)$, all the momentum matrix elements are equal : $P_{S, x}=P_{S, y}=$ $P_{S, z}$ (see Appendix B). In bulk or in a shape-isotropic NC $\left(L_{x}=L_{y}=L_{z}\right)$, the three bright exciton states are degenerate and there is no splitting. In a $\mathrm{NC}$ (either in the strong or weak confinement regime), an anisotropy along one single direction $\left(L_{y}=L_{z} \neq L_{x}\right)$ will induce a partial degeneracy lift and the appearance of a doublet splitting $\Delta E$. This splitting is shown in Fig. 2 (green lines), for the different compounds and a fixed shape anisotropy : $r=s=0.9$ with $r=L_{y} / L_{x}$ and $s=L_{z} / L_{x}$ (the other parameters are given in Table I). In the weak confinement regime, one observes an increase of $\Delta E$ from iodide $(75.2 \mu \mathrm{eV})$ to bromide $(219 \mu \mathrm{eV})$, then chloride $(506 \mu \mathrm{eV})$. This increase is mainly related to the $a_{X}^{-3}$ dependence of the e-h EI. In the strong confinement regime, for a given NC size, the splitting is no more $a_{X}$-dependent, but is proportional to $\left(E_{P_{S, \rho}} / E_{g}^{2} \epsilon_{r}\right)$ (almost constant for the three

compounds $\left.\left(E_{P_{S, \rho}} / E_{g}^{2} \epsilon_{r}\right)=(1.01 \pm 0.22) \mathrm{eV}^{-1}\right)$, which explains comparable splitting values for the three compounds, for small NC. In a NC of highly anisotropic shape, a full splitting of the bright excitonic level into a triplet $|Y\rangle,|X\rangle$, and $|Z\rangle$ is obtained. The energy splitting of the triplets are shown in Fig. 2 (red and blue lines) with a shape anisotropy $r=L_{y} / L_{x}=0.9$ and $s=L_{z} / L_{x}=1.1$. The splittings $\Delta E_{1}$ and $\Delta E_{2}$ are inversely proportional to the NC volume. For this particular set of parameters, $\Sigma_{3}<\Sigma_{2}<\Sigma_{1}$, respectively associated to the $|Z\rangle,|X\rangle$ and $|Y\rangle$ states. Note that this order can be changed with the parameters $(r, s)$ and then Fig. 1a will be modified. 

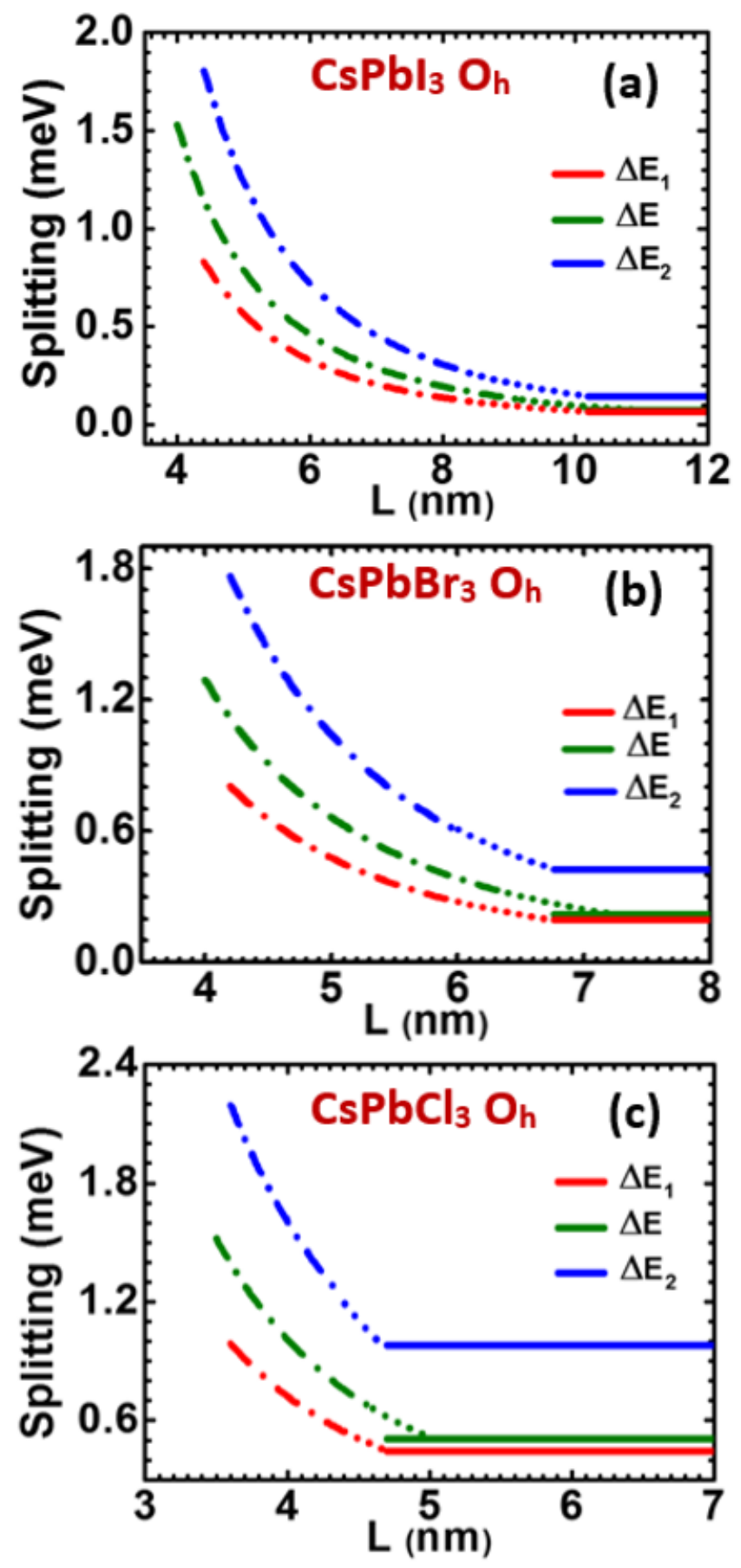

Fig. 2: Bright-exciton splitting due to NC anisotropy calculated in four-band $\mathbf{k} \cdot \mathbf{p}$ model for three perovskite materials as a function of $N C$ size in the cubic symmetry $\left(O_{h}\right)$, assuming $10 \%$ shape anisotropy of the NC. Solid lines show the weak confinement regime, the dash-dotted lines the strong confinement regime, and the dashed lines (linking the two regimes) are guides to the eye. Doublet splitting $\Delta E$ is calculated for $r=s=0.9$. Triplet splittings $\Delta E_{1}$ and $\Delta E_{2}$ are calculated for $r=0.9$ and $s=1.1$. 
In the tetragonal symmetry $\left(D_{4 h}\right)$, the three bright exciton states are no more fully degenerate, in a bulk or in a shape-isotropic NC; this intrinsic asymmetry induces a partial degeneracy lift.

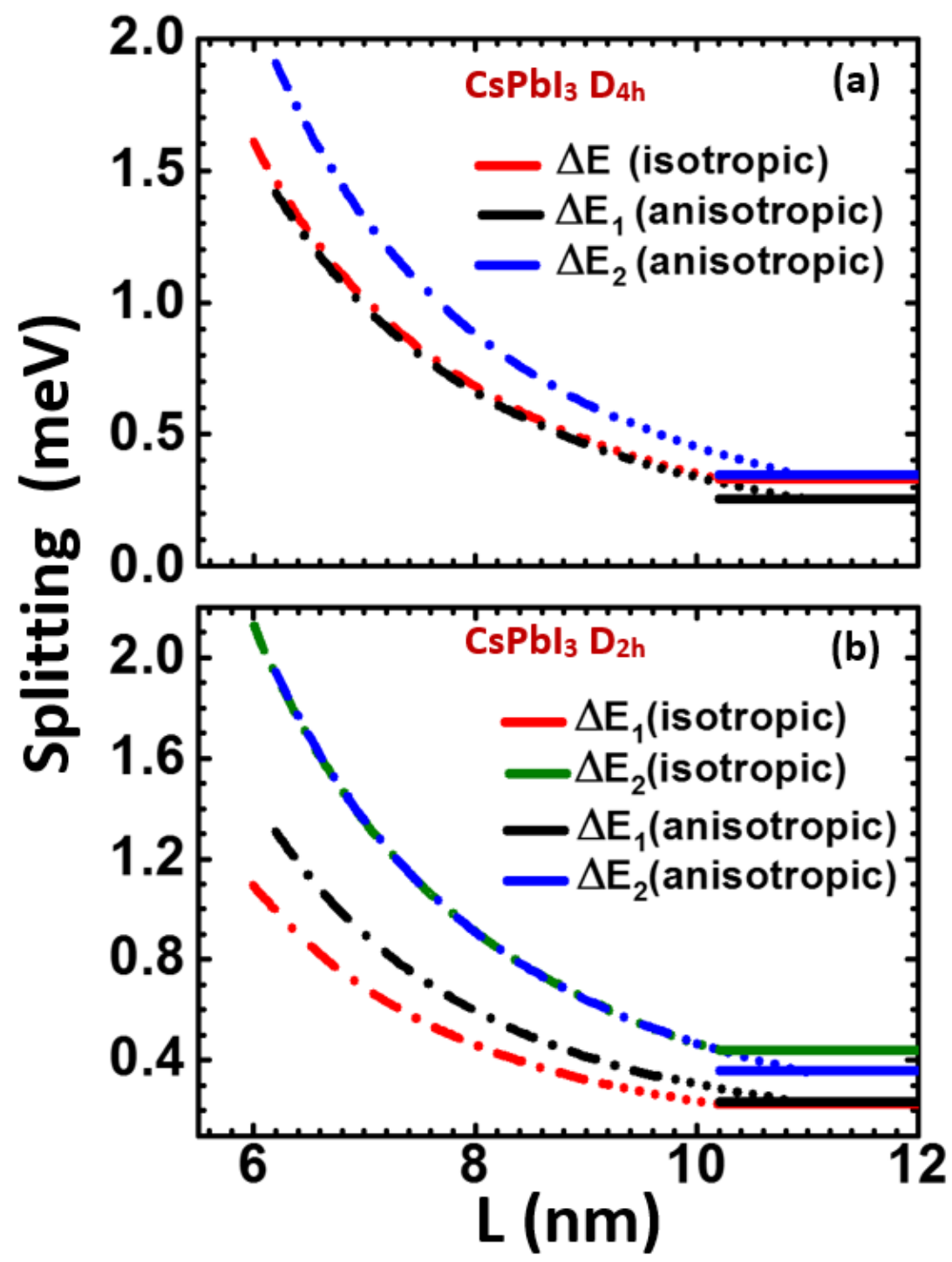

Fig. 3: Bright-exciton splitting, due to both intrinsic and extrinsic properties, calculated in the four-band k.p model for $\mathrm{CsPbI}_{3}$ as a function of $\mathrm{NC}$ size : (a) in the tetragonal symmetry $\left(D_{4 h}\right)$, (b) in the orthorhombic symmetry $\left(D_{2 h}\right)$. "Anisotropic" refers to a fixed anisotropy of $10 \%$ for NC shape $(r=s=0.9)$. Solid lines show the weak confinement regime, the dash-dotted lines the strong confinement regime, and the dashed lines are guides to the eye.

There are two contributions, caused by the tetragonal crystal field. The main one is associated to the change in the CB wave functions, related to the parameter $\theta$ (see Appendix 
A). The second contribution to the exciton splitting is due to the anisotropic momentum matrix elements: $P_{S, x}=P_{S, y} \neq P_{S, z}$ (see Appendix B). The splitting $\Delta E$ is shown in Figs. 3a, 4a and 5a (red lines), for the different compounds $\mathrm{CsPbX}_{3}, X=I, \mathrm{Br}$ and $\mathrm{Cl}$, respectively. We have assumed a $10 \%$ anisotropy between the momentum matrix elements (see Table I).

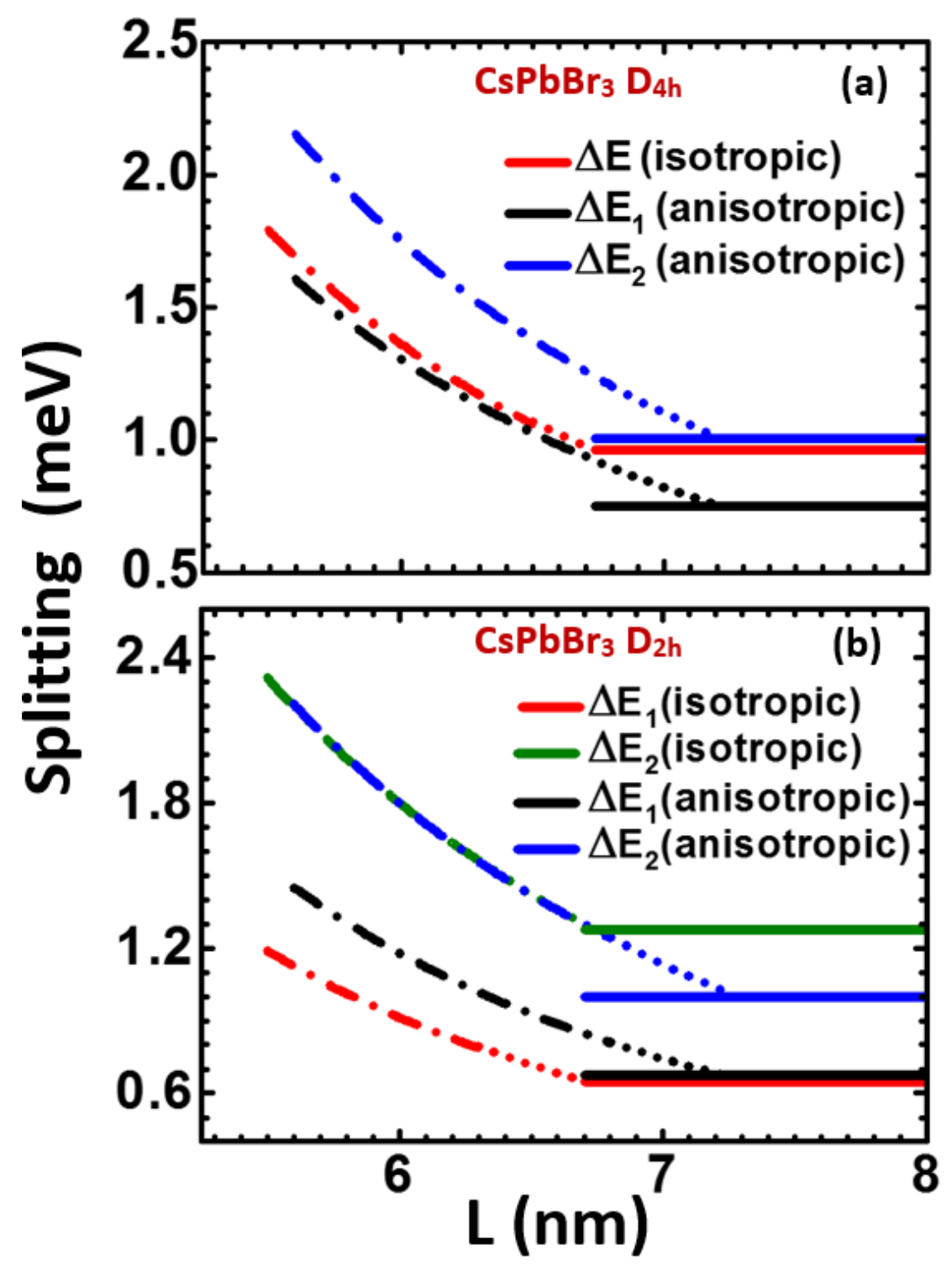

Fig. 4: Bright-exciton splitting, due to both intrinsic and extrinsic properties, calculated in the four-band k.p model for $\mathrm{CsPbBr}$ as a function of $\mathrm{NC}$ size: (a) in the tetragonal symmetry $\left(D_{4 h}\right),(b)$ in the orthorhombic symmetry $\left(D_{2 h}\right)$. "Anisotropic" refers to a fixed anisotropy of $10 \%$ for $N C$ shape $(r=s=0.9)$. Solid lines show the weak confinement regime, the dash-dotted lines the strong confinement regime, and the dashed lines are guides to the eye. 


\begin{tabular}{cllc}
\hline \hline & $D_{4 h}$ & \multicolumn{2}{c}{$D_{2 h}$} \\
\hline$m e V$ & $\Delta E$ & $\Delta E_{1}$ & $\Delta E_{2}$ \\
\hline $\mathrm{CsPbI}_{3}$ & 0.33 & 0.22 & 0.44 \\
$\mathrm{CsPbBr}_{3}$ & 0.96 & 0.65 & 1.27 \\
$\mathrm{CsPbCl}_{3}$ & 2.21 & 1.5 & 2.95 \\
\hline \hline
\end{tabular}

TABLE II: Calculated exchange splittings for the bulk $\mathrm{CsPbX}_{3}(X=C l, B r, I)$ for both tetragonal and orthorhombic symetries.

As in the cubic symmetry $\left(O_{h}\right)$, in the weak confinement regime, one observes an increase of $\Delta E$ from iodide $(331 \mu \mathrm{eV})$ to bromide $(962 \mu \mathrm{eV})$, then chloride $(2.21 \mathrm{meV})$ due to the $a_{X}$ dependence. Once again, in the strong confinement regime, the splitting values are comparable for the three halide perovskite NC. When the $\mathrm{NC}$ shape is anisotropic along one direction, the bright excitonic levels are completely splitted, forming a triplet $|Y\rangle,|X\rangle$, and $|Z\rangle$.

The energy splitting of the triplets, $\Delta E_{1}$ and $\Delta E_{2}$, are shown in Figs. 3a, 4a and 5a (black and blue lines) with a shape anisotropy $r=s=0.9$. This shape anisotropy increases slightly the level splittings: $\Delta E_{1}<\Delta E<\Delta E_{2}$. Once again, for this particular shape anisotropy $(r=s=0.9)$, the eigenstates are $|Y\rangle,|X\rangle$, and $|Z\rangle$, with decreasing energy (this may vary with the values of $r$ and $\theta$ ). We have verified that the calculated $\Delta E$ values with $L_{x}=L_{y}=L_{z}$ in the $D_{4 h}$ symmetry for the weak confinement regime are the same as the computed ones in the bulk which are given in Table II.

In the orthorhombic symmetry $\left(D_{2 h}\right)$, the bright excitonic levels are completely splitted, in a shape-isotropic NC. While in the tetragonal symmetry $\left(D_{4 h}\right)$, the conduction states present equal weight in the $X_{C}$ and $Y_{C}$ Bloch components, the situation is different in the $D_{2 h}$ symmetry, due to the orthorhombic crystal field ( $\alpha \neq \beta$, see Appendix A).

Due to the previous contributions (tetragonal crystal field, anisotropic momentum matrix elements), one has then a full degeneracy splitting. The splittings $\Delta E_{1}$ and $\Delta E_{2}$ are shown in Figs. 3b, 4b and 5b (red and green lines), for the different compounds and an isotropic NC shape. We have assumed the same anisotropy of the momentum matrix elements, as for $D_{4 h}$ (neglecting the $\mathrm{x}-\mathrm{y}$ anisotropy). As in the cubic symmetry $\left(O_{h}\right)$, in the weak confinement regime, one observes an increase of $\Delta E_{1}$ and $\Delta E_{2}$ from iodide, to bromide, then chloride. Once again, in the strong confinement regime, the splitting values are comparable for the three halide perovskite NC. 
For comparison, we have also calculated the size-dependence of the splitting for an anisotropic NC, along one direction $r=s=0.9$ (see Figs. 3b, 4b and 5b; black and blue lines), with a significant change for $\Delta E_{1}$, while $\Delta E_{2}$ is almost unchanged.

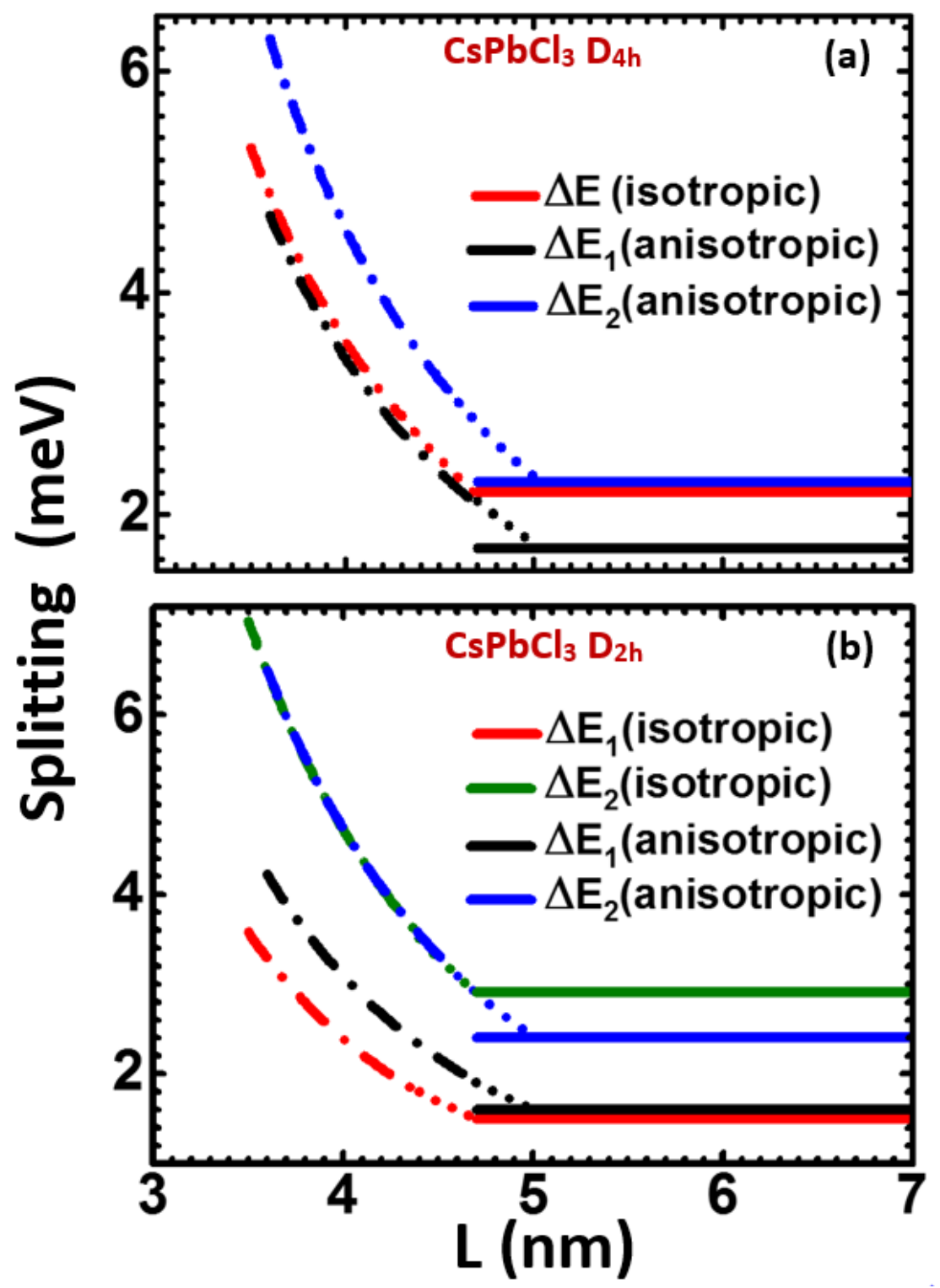

Fig. 5: Bright-exciton splitting, due to both intrinsic and extrinsic properties, calculated in the four-band k.p model for $\mathrm{CsPbCl}_{3}$ as a function of $\mathrm{NC}$ size : (a) in the tetragonal symmetry $\left(D_{4 h}\right)$, (b) in the orthorhombic symmetry $\left(D_{2 h}\right)$. "Anisotropic" refers to a fixed anisotropy of $10 \%$ for $N C$ shape $(r=s=0.9)$. Solid lines show the weak confinement regime, the dash-dotted lines the strong confinement regime, and the dashed lines are guides to the eye.

Let us recall that the behaviours of "anisotropic" $\Delta E_{1}$ and $\Delta E_{2}$ have been computed here 
in the particular anisotropy conditions, namely $r=s=0.9$. Note that $\Delta E_{2}$ is more sensitive upon $r$ than $\Delta E_{1}$. Finally, in the $D_{2 h}$ symmetry, we have also checked that the calculated isotropic $\Delta E_{1}$ and $\Delta E_{2}$ values with $L_{x}=L_{y}=L_{z}$ in the weak confinement regime are equal to the bulk computed values listed in Table II. In Fig. 6, we plot the exchange splittings as a function of $a_{X}^{-3}$ and one observes a linearly dependence obtained for $\Delta E$ in $D_{4 h}$ and $\left(\Delta E_{1}, \Delta E_{2}\right)$ in $D_{2 h}$, indicating that in the weak confinement regime, the splitting is mainly related on the $a_{X}^{-3}$ dependence.

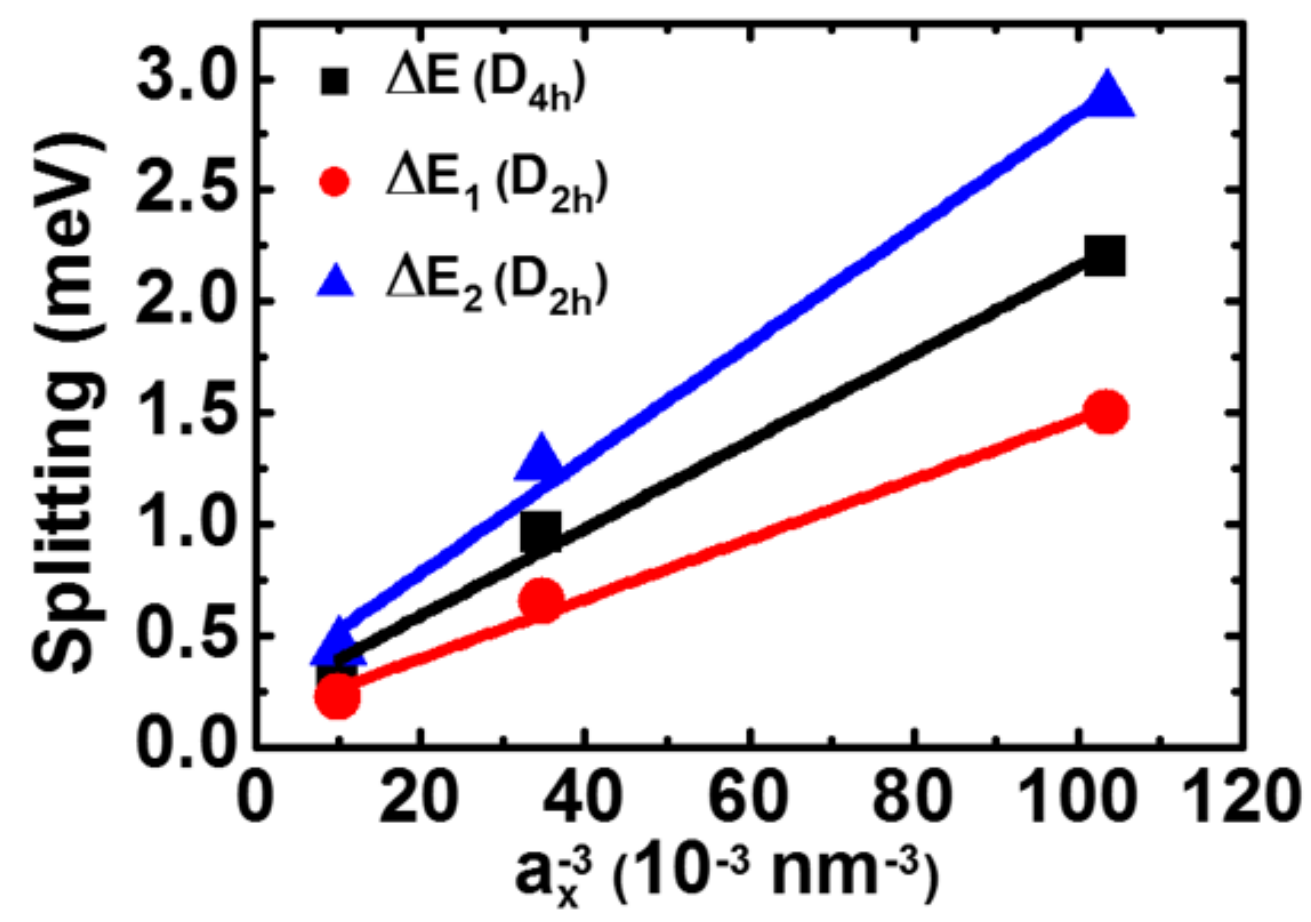

Fig. 6: The dependence of the splitting on the $a_{X}^{-3}$ in the weak confinement regime.

Few experimental PL studies on a single NC have been performed up to now. Recent results obtained in pure (unalloyed) perovskite compounds are presented in Table III, with doublet or triplet splittings, and the average NC size and exciton emission. Table III shows also results concerning alloyed NCs. The comparison with theoretical results are more complicated for the latter cases. However, large splitting have been observed by Becker et al [21] who have invoked Rashba effects in distorted orthorhombic lattices. Note that for this alloyed compound $\mathrm{CsPbBr}_{3-x} \mathrm{Cl}_{x}$, the measured splittings are intermediate between our calculated splittings for $\mathrm{CsPbBr}_{3}$ and $\mathrm{CsPbCl}_{3}$.

Concerning non-alloyed $\mathrm{CsPbBr}_{3}$ and $\mathrm{CsPbI}_{3} \mathrm{NCs}$, we observe for comparable $\mathrm{NC}$ sizes, a larger doublet splitting $\Delta E$ for the bromide than for Iodide compound, in agreement with 


\begin{tabular}{|c|c|c|c|c|c|c|}
\hline Reference & Compound & $\begin{array}{l}<L> \\
(\mathrm{nm})\end{array}$ & $\begin{array}{c}\Delta E \\
(\mathrm{meV})\end{array}$ & $\begin{array}{l}\Delta E_{1} \\
(\mathrm{meV})\end{array}$ & $\begin{array}{c}\Delta E_{2} \\
(\mathrm{meV})\end{array}$ & $\begin{array}{c}\text { Emission energy } \\
(\mathrm{eV})\end{array}$ \\
\hline Ramade $e t a l^{a}$ & $\mathrm{CsPbBr}_{3}$ & 11.5 & $1.00 \pm 0.20$ & $0.50 \pm 0.20$ & $1.0 \pm 0.2$ & $2.47-2.51$ \\
\hline $\mathrm{Fu}$ et $a l^{b}$ & $\mathrm{CsPbBr}_{3}$ & 9.0 & 1.00 & 0.60 & 1.0 & $2.39-2.45$ \\
\hline Yin et $a l^{c}$ & $\mathrm{CsPbI}_{3}$ & 9.3 & 0.40 & & & $1.70-1.75$ \\
\hline Nestoklon et $a l^{d}$ & $\mathrm{CsPbI}_{3}$ & 10.0 & 0.12 & & & 1.72 \\
\hline Raino et $a l^{e}$ & $\mathrm{CsPb}(\mathrm{Cl} / \mathrm{Br})_{3}$ & 9.5 & & 0.75 & 1.5 & 2.48 \\
\hline Becker et al ${ }^{f}$ & $\mathrm{CsPbBr}_{2} \mathrm{Cl}$ & $14.0 \pm 1.0$ & 1.63 & 1.07 & 2.3 & $2.5-2.6$ \\
\hline
\end{tabular}

${ }^{a}$ Reference 19.

${ }^{b}$ Reference 18.

${ }^{c}$ Reference 22.

${ }^{d}$ Reference 54 .

${ }^{e}$ Reference 9 .

${ }^{f}$ Reference 21 .

TABLE III: Summarized experimental data, namely the bright-exciton splittings and the emission energy, from literature.

the theoretical prediction. For $\mathrm{CsPbI}_{3}$, Yin et al. [22] have observed a doublet splitting $\Delta E=0.4 \pm 0.1 \mathrm{meV}(<L>=9.3 \mathrm{~nm})$. This value compares well with our calculation for $D_{4 h}$ crystal and isotropic shape (Fig. 3a ; $\Delta E=0.422 \mathrm{meV}$ for $\langle L\rangle=9.4 \mathrm{~nm}$ ). More recently, Nestoklon et al. [54] have measured over an ensemble doublet splitting $\Delta E=120$ $\mu \mathrm{eV}$, in $\mathrm{NC}$ with a cubic symmetry $\left(O_{h}\right)$ and an anisotropic shape $(<L>=10 \mathrm{~nm} ; 10 \%$ anisotropy). This has to be compared with our theoretical value $99 \mu \mathrm{eV}$ (Fig. 2a) in $O_{h}$ symmetry (and $10 \%$ anisotropy), or with $\Delta E=331 \mu \mathrm{eV}$ in $D_{4 h}$ symmetry (isotropic NC).

For $\mathrm{CsPbBr}_{3} \mathrm{NC}$, the doublet splitting is typically $\Delta E=1 \mathrm{meV}$, while the triplet splitting are $\Delta E_{1} \simeq 0.6 \mathrm{meV}$ and $\Delta E_{2} \simeq 1.0 \mathrm{meV}$, for $\langle L\rangle=9.5-11.5 \mathrm{~nm}[18,19]$. These triplet splittings are comparable to calculation for isotropic NC in the orthorhombic phase (see Fig. 4b; 0.649 and $1.276 \mathrm{meV}$, in the weak confinement regime).

\section{CONCLUSION}

The contribution of the long range electron-hole exchange interaction, for the bright exciton splittings, in $\mathrm{CsPbX}_{3}(X=C l, B r, I)$ NCs is studied using the group theory and k.p arguments. We show that the exciton fine structure is the result of an interplay of the shape and the crystalline phase anisotropies of inorganic halide perovskite NCs. The calculated exchange magnitudes agree fairly well with experimental results and set up a suitable model correlating the observed bright-exciton splittings to specific perovskite NCs 
characteristics (size, composition, crystal structure, anisotropic shape). That provides valuable informations to seek the possibillity of realizing nanophotonic quantum devices based on the manipulation of the bright-exciton fine structure.

NCs with $O_{h}$ crystallographic phase and isotropic shape show a single PL line, but triplet PL lines can also be observed experimentally in highly shape anisotropic NCs. In tetragonal symmetry, a simple shape anisotropy is sufficient to lead to a triplet state, while in orthorhombic symmetry, no shape anisotropy is necessary. Moreover, if we take into account thermal population for NCs in strong confinement regime, it will be also possible to observe experimentally, for $D_{4 h}$ and $D_{2 h}$ phases, single lines in the photoluminescence spectra of single NCs when the thermal energy is lower than the splitting energy of the upper excitonic levels. Finally, in order to account for both influences of crystal structure and shape anisotropy of a single NC on its emission characteristics, the correlation between observations in electronic and luminescence microscopies and X-ray diffraction represents striking and pertinent further investigations.

\section{Acknowledgments}

This work was partially supported by the French National Research Agency (ANR IPERNano2, ANR-18-CE30-0023-01). 


\section{APPENDIX A: BLOCH FUNCTIONS}

This appendix outlines the basis Bloch wave functions of the upper VB and the lowest split-off CB. The top of the VB arises from the cationic s-orbitals resulting from the overlap between metal s-orbitals and halide p-orbitals, with an overall s symmetry. We denote the hole Bloch wave functions as

$$
\left|W_{1}\right\rangle=\left|S_{V} \uparrow\right\rangle \quad ; \quad\left|W_{2}\right\rangle=\left|S_{V} \downarrow\right\rangle
$$

where $\uparrow(\downarrow)$ denotes the spin-up (down) state and $\left|S_{V}\right\rangle$ represents the s-like VB Bloch wave functions.

The CB arises from the metal p-orbitals, leading to three possible orthogonal spatial components for the Bloch wave functions, namely $\left|X_{C}\right\rangle,\left|Y_{C}\right\rangle$ or $\left|Z_{C}\right\rangle$. The reduction of the lattice point symmetry from cubic $\left(O_{h}\right)$ to tetragonal $\left(D_{4 h}\right)$ or orthorhombic $\left(D_{2 h}\right)$ leads to an additional term in the Hamiltonian due to the crystal field. Consequently, from one group to another, the CB Bloch wave functions do not have the same expressions.

The Bloch wave functions for the irreductible representation $\Gamma_{6}^{-}$of the group $D_{4 h}$, which corresponds to the lowest $\mathrm{CB}$, are given by

$$
\begin{aligned}
& \left|C_{1}\right\rangle=-i\left[\frac{\cos \theta}{\sqrt{2}}\left|\left(X_{C}+i Y_{C}\right) \downarrow\right\rangle+\sin \theta\left|Z_{C} \uparrow\right\rangle\right] \\
& \left|C_{2}\right\rangle=i\left[-\frac{\cos \theta}{\sqrt{2}}\left|\left(X_{C}-i Y_{C}\right) \uparrow\right\rangle+\sin \theta\left|Z_{C} \downarrow\right\rangle\right]
\end{aligned}
$$

in which $\tan 2 \theta=2 \sqrt{2} \Delta /(\Delta-3 T)$ (with $0<\theta<\pi / 2), \Delta$ is the spin-orbit splitting of the $\mathrm{CB}$ and $T$ is the additional tetragonal crystal field term.

The phases of these functions are chosen to yield the Bloch wave functions of the representation $\Gamma_{6}^{-}$of the group $O_{h}$ in the limit $T=0$, thus leads to take the values $\cos \theta=\sqrt{2 / 3}$ and $\sin \theta=\sqrt{1 / 3}$ in Eqs. ((A2)-(A3)).

For the $D_{2 h}$ case, an additional crystal field term, $\epsilon$, is required to account the symmetry lowering in the orthorhombic structure. Following the development given in Ref. [18], we write the lowest CB states as 


$$
\begin{aligned}
& \left|C_{1}\right\rangle=i\left[-\alpha\left|X_{C} \downarrow\right\rangle-i \beta\left|Y_{C} \downarrow\right\rangle+\gamma\left|Z_{C} \uparrow\right\rangle\right] \\
& \left|C_{2}\right\rangle=i\left[-\alpha\left|X_{C} \uparrow\right\rangle+i \beta\left|Y_{C} \uparrow\right\rangle-\gamma\left|Z_{C} \downarrow\right\rangle\right]
\end{aligned}
$$

with $\alpha^{2}+\beta^{2}+\gamma^{2}=1$. The real constants $(\alpha, \beta, \gamma)$ are given explicitly in Ref. [19]. They depend on $\epsilon, \theta$, and the bottom energies of the different CBs. The lowest CB states of the $D_{2 h}$ case tend towards the ones of the $D_{4 h}$ group in the limit $\epsilon \rightarrow 0$.

In Appendix B, the states $\left|W_{1}\right\rangle,\left|W_{2}\right\rangle,\left|C_{1}\right\rangle$, and $\left|C_{2}\right\rangle$ will be used to obtain the matrix elements of the LR e-h EI.

\section{APPENDIX B: MATRIX REPRESENTATION OF LONG-RANGE EXCHANGE}

\section{INTERACTION}

The point-group symmetry provides a standard systematic approach to construct the

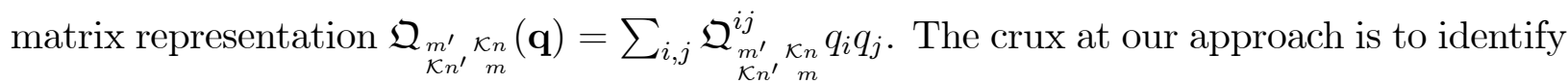
the nonzero matrix elements of the operator $\mathcal{H}_{\mathbf{k} . \mathbf{p}}=\frac{\hbar}{m_{0}} \mathbf{k} \cdot \mathbf{p}$, where $m_{0}$ is the free electron mass. For this purpose, we must use the appropriate irreductible representation for the momentum operator $\mathbf{p}$ for each point group. Indeed, due to lattice or shape distortions, the irreductible representation of operator $\mathbf{p}$ is modified when we descend in symmetry from cubic $\left(O_{h}\right)$ to tetragonal $\left(D_{4 h}\right)$ or orthorhombic $\left(D_{2 h}\right)$.

\section{Orthorhombic phase $D_{2 h}$}

Under $D_{2 h}$ operations, the components $p_{x}, p_{y}, p_{z}$ transform like $\Gamma_{4}^{-}, \Gamma_{2}^{-}, \Gamma_{3}^{-}$, respectively. Moreover, we have $\left(X_{C}, Y_{C}, Z_{C}\right) \sim\left(\Gamma_{4}^{-}, \Gamma_{2}^{-}, \Gamma_{3}^{-}\right)$and $S_{V} \sim \Gamma_{1}^{+}$. The symbol " $"$ tells us how these orbital functions transform under $\mathrm{D}_{2 h}$ operations. Note that $S_{V}$ remains $\sim \Gamma_{1}^{+}$ in the different phases: cubic $\left(O_{h}\right)$, tetragonal $\left(D_{4 h}\right)$ and orthorhombic $\left(D_{2 h}\right)$. According to the point group symmetry and k.p arguments, the nonzero matrix elements constructed from the momentum operator $\mathbf{p}$ are $P_{S, x}=\frac{\hbar}{m_{0}}\left\langle S_{V}\left|p_{x}\right| i X_{C}\right\rangle, P_{S, y}=\frac{\hbar}{m_{0}}\left\langle S_{V}\left|p_{y}\right| i Y_{C}\right\rangle, P_{S, z}=$ $\frac{\hbar}{m_{0}}\left\langle S_{V}\left|p_{z}\right| i Z_{C}\right\rangle$. All these k.p matrix elements are real parameters. 
With the basis $\left|C_{m} W_{n}\right\rangle$ taken in the order $\left\{\begin{array}{c}\left|C_{1} W_{1}\right\rangle=\left|j_{z}^{e}=1 / 2, j_{z}^{h}=1 / 2\right\rangle,\left|C_{2} W_{1}\right\rangle=|-1 / 2,1 / 2\rangle, \\ \left|C_{1} W_{2}\right\rangle=|1 / 2,-1 / 2\rangle,\left|C_{2} W_{2}\right\rangle=|-1 / 2,-1 / 2\rangle\end{array}\right\}$ the matrix $\mathfrak{Q}_{\substack{m^{\prime} n^{\prime} \\ \mathcal{K}_{n} \\ m}}(\mathbf{q})$ takes the form:

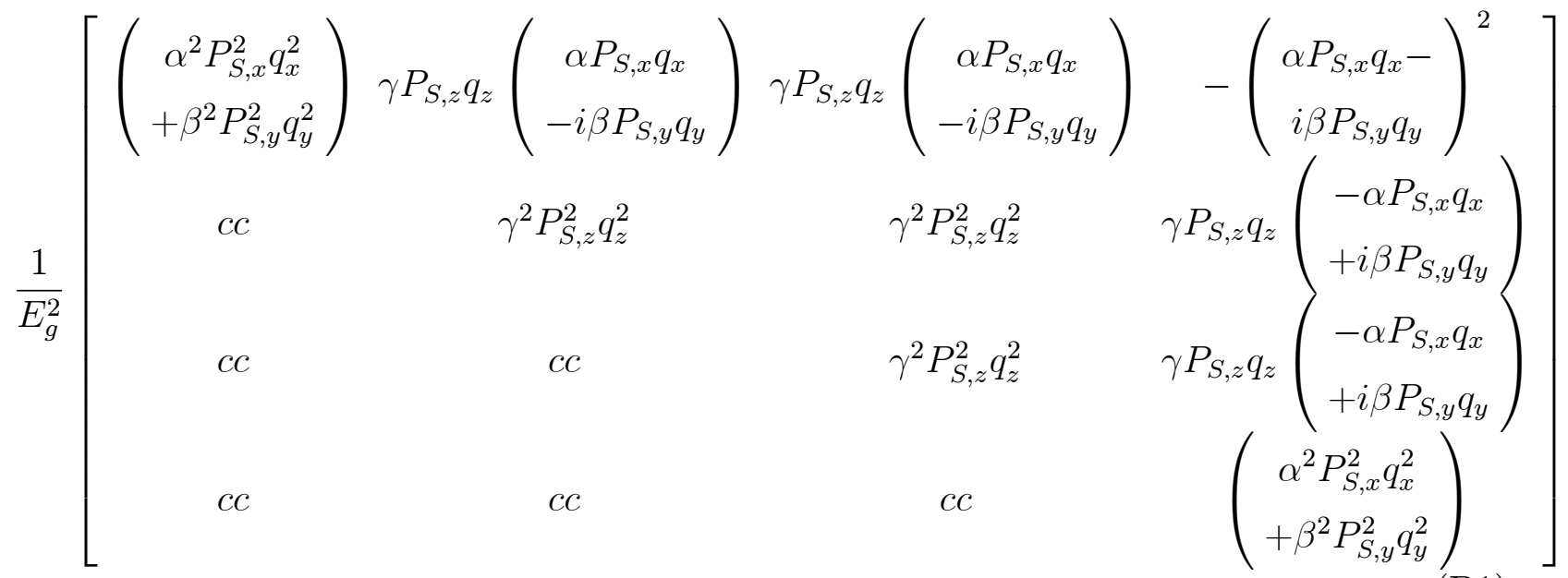

where $E_{g}$ is the band-gap energy and $c c$ denotes the complex conjugate. Let us introduce the bright triplet states $\left(j=j_{e}+j_{h}=1\right),|+1\rangle,\left|O_{B}\right\rangle,|-1\rangle$, and the dark singlet state $(j=0)$, $\left|O_{D}\right\rangle:$

$$
\left\{\begin{array}{c}
|+1\rangle=\left|j_{z}^{e}=1 / 2, j_{z}^{h}=1 / 2\right\rangle \\
\left|O_{B}\right\rangle=\frac{1}{\sqrt{2}}(|1 / 2,-1 / 2\rangle+|-1 / 2,1 / 2\rangle) \quad ; \quad\left|O_{D}\right\rangle=\frac{1}{\sqrt{2}}(|1 / 2,-1 / 2\rangle-|-1 / 2,1 / 2\rangle) \\
|-1\rangle=|-1 / 2,-1 / 2\rangle
\end{array}\right.
$$

In the basis $\left\{|+1\rangle,|-1\rangle,\left|O_{B}\right\rangle ;\left|O_{D}\right\rangle\right\}$, we can rewriting the LR e-h EI matrix as:

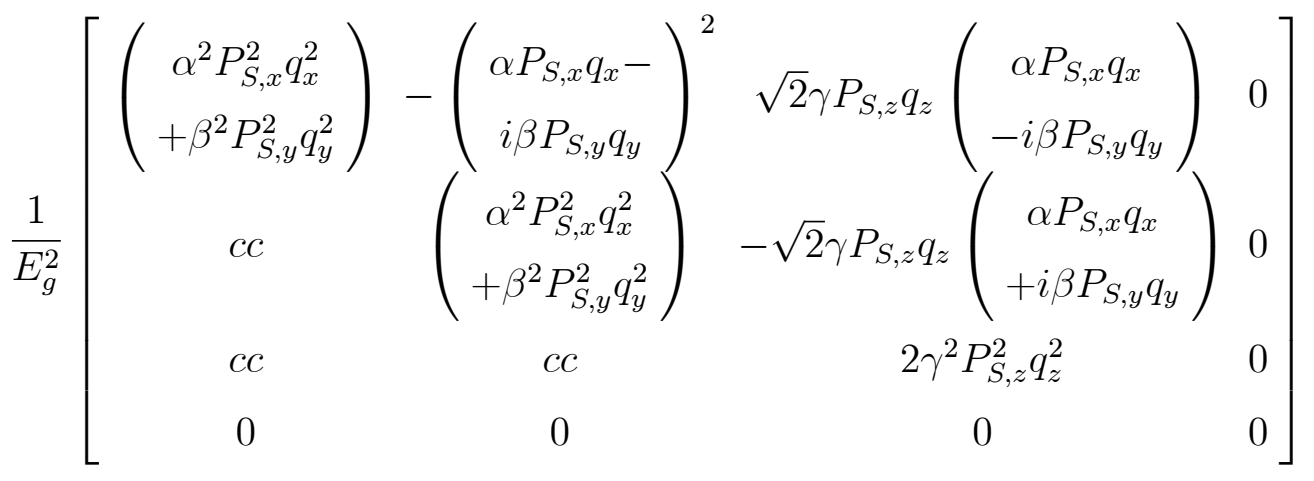


Using the Fourier transform of the exciton envelope function, $\Psi_{X}^{\alpha}\left(\mathbf{r}_{e}, \mathbf{r}_{h}\right)(\alpha=s c, w c, b)$, with coinciding electron and hole coordinates, and substituting its square into Eq. (1), we get the LR matrix representation $\mathcal{H}_{\text {exch }}^{L R}$ (see Eq. (6)) in which $\left(\Sigma_{d}, \Sigma_{o d}, \Sigma_{z}\right)$ are given by the following relationships:

$$
\left\{\begin{array}{c}
\Sigma_{d}=\frac{\alpha^{2}}{E_{g}^{2}}\left(\frac{\hbar^{2}}{2 m_{0}} E_{P_{S, x}}\right) \int d \mathbf{q} q_{x}^{2} \mathcal{V}_{q}\left|\int d \mathbf{r} \Psi_{X}^{\alpha} \exp (i \mathbf{q} \cdot \mathbf{r})\right|^{2} \\
+\frac{\beta^{2}}{E_{g}^{2}}\left(\frac{\hbar^{2}}{2 m_{0}} E_{P_{S, y}}\right) \int d \mathbf{q} q_{y}^{2} \mathcal{V}_{q}\left|\int d \mathbf{r} \Psi_{X}^{\alpha} \exp (i \mathbf{q} \cdot \mathbf{r})\right|^{2} \\
\Sigma_{o d}=-\frac{\alpha^{2}}{E_{g}^{2}}\left(\frac{\hbar^{2}}{2 m_{0}} E_{P_{S, x}}\right) \int d \mathbf{q} q_{x}^{2} \mathcal{V}_{q}\left|\int d \mathbf{r} \Psi_{X}^{\alpha} \exp (i \mathbf{q} \cdot \mathbf{r})\right|^{2} \\
\quad+\frac{\beta^{2}}{E_{g}^{2}}\left(\frac{\hbar^{2}}{2 m_{0}} E_{P_{S, y}}\right) \int d \mathbf{q} q_{y}^{2} \mathcal{V}_{q}\left|\int d \mathbf{r} \Psi_{X}^{\alpha} \exp (i \mathbf{q} \cdot \mathbf{r})\right|^{2} \\
\Sigma_{z}=\frac{2 \gamma^{2}}{E_{g}^{2}}\left(\frac{\hbar^{2}}{2 m_{0}} E_{P_{s, z}}\right) \int d \mathbf{q} q_{z}^{2} \mathcal{V}_{q}\left|\int d \mathbf{r} \Psi_{X}^{\alpha} \exp (i \mathbf{q} . \mathbf{r})\right|^{2}
\end{array}\right.
$$

where $\mathcal{V}_{q}=\frac{1}{(2 \pi)^{3}}\left(\frac{e^{2}}{\epsilon_{0} \epsilon_{X}}\right) \frac{1}{q^{2}}$ is the Fourier transform of the Coulomb potential. We define the related energies as usual, namely the energies $E_{P_{S, \ell}}(\ell=x, y, z)$ are defined by $E_{P_{S, \ell}}=$ $\left(2 m_{0} / \hbar^{2}\right) P_{S, l}^{2}$.

\section{Tetragonal phase $D_{4 h}$}

Under $D_{4 h}$ operations, the two transverse components $\left(p_{x}, p_{y}\right)$ transform like $\Gamma_{5}^{-}$while $p_{z}$ transforms such as $\Gamma_{2}^{-}$. Contrary to the upper VB, the lowest split-off CB no longer belongs the same irreductible representation. We have $\left(X_{C}, Y_{C}\right) \sim \Gamma_{5}^{-}$and $Z_{C} \sim \Gamma_{2}^{-}$. Only two momentum matrix elements can be constructed. We denote them by $P_{S, \rho}=\frac{\hbar}{m_{0}}\left\langle S_{V}\left|p_{x}\right| i X_{C}\right\rangle=$ $\frac{\hbar}{m_{0}}\left\langle S_{V}\left|p_{y}\right| i Y_{C}\right\rangle$ and $P_{S, z}=\frac{\hbar}{m_{0}}\left\langle S_{V}\left|p_{z}\right| i Z_{C}\right\rangle$. Following the usual procedure and taking for the tetragonal symmetry, $\alpha=\beta=(\cos \theta) / \sqrt{2}, \gamma=-\sin \theta, P_{S, x}=P_{S, y}=P_{S, \rho}$, we can deduce $\mathcal{H}_{\text {exch }}^{L R}$. The nonzero matrix elements of $\mathcal{H}_{\text {exch }}^{L R},\left(\Sigma_{d}, \Sigma_{o d}, \Sigma_{z}\right)$, can now be written as

$$
\left\{\begin{array}{c}
\Sigma_{d}=\frac{1}{2 E_{g}^{2}}\left(\frac{\hbar^{2}}{2 m_{0}} E_{P_{S, \rho}}\right)\left(\cos ^{2} \theta\right) \int d \mathbf{q} q_{\rho}^{2} \mathcal{V}_{q}\left|\int d \mathbf{r} \Psi_{X}^{\alpha} \exp (i \mathbf{q} \cdot \mathbf{r})\right|^{2} \\
\Sigma_{o d}=\frac{1}{2 E_{g}^{2}}\left(\frac{\hbar^{2}}{2 m_{0}} E_{P_{S, \rho}}\right)\left(\cos ^{2} \theta\right) \int d \mathbf{q}\left(q_{x}^{2}-q_{y}^{2}\right) \mathcal{V}_{q}\left|\int d \mathbf{r} \Psi_{X}^{\alpha} \exp (i \mathbf{q} \cdot \mathbf{r})\right|^{2} \\
\Sigma_{z}=\frac{2}{E_{g}^{2}}\left(\frac{\hbar^{2}}{2 m_{0}} E_{P_{S, z}}\right)\left(\sin ^{2} \theta\right) \int d \mathbf{q} q_{z}^{2} \mathcal{V}_{q}\left|\int d \mathbf{r} \Psi_{X}^{\alpha} \exp (i \mathbf{q} \cdot \mathbf{r})\right|^{2}
\end{array}\right.
$$

where $q_{\rho}^{2}=\left(q_{x}^{2}+q_{y}^{2}\right)$ and $E_{P_{S, \ell}}=\left(2 m_{0} / \hbar^{2}\right) P_{S, \ell}^{2}(\ell=\rho, z)$. 


\section{Cubic phase $O_{h}$}

In the cubic system, we have $\cos \theta=\sqrt{2 / 3}, \sin \theta=\sqrt{1 / 3}, P_{S, \rho}=P_{S, z}=P_{S}=$ $\frac{\hbar}{m_{0}}\left\langle S_{V}\left|p_{x}\right| i X_{C}\right\rangle=\frac{\hbar}{m_{0}}\left\langle S_{V}\left|p_{y}\right| i Y_{C}\right\rangle=\frac{\hbar}{m_{0}}\left\langle S_{V}\left|p_{z}\right| i Z_{C}\right\rangle$, and the nonzero matrix elements of $\mathcal{H}_{\text {exch }}^{L R}$ become

$$
\left\{\begin{array}{c}
\Sigma_{d}=\frac{1}{3 E_{g}^{2}}\left(\frac{\hbar^{2}}{2 m_{0}} E_{P_{S}}\right) \int d \mathbf{q}\left(q_{x}^{2}+q_{y}^{2}\right) \mathcal{V}_{q}\left|\int d \mathbf{r} \Psi_{X}^{\alpha} \exp (i \mathbf{q} . \mathbf{r})\right|^{2} \\
\Sigma_{o d}=\frac{1}{3 E_{g}^{2}}\left(\frac{\hbar^{2}}{2 m_{0}} E_{P_{S}}\right) \int d \mathbf{q}\left(q_{x}^{2}-q_{y}^{2}\right) \mathcal{V}_{q}\left|\int d \mathbf{r} \Psi_{X}^{\alpha} \exp (i \mathbf{q} \cdot \mathbf{r})\right|^{2} \\
\Sigma_{z}=\frac{2}{3 E_{g}^{2}}\left(\frac{\hbar^{2}}{2 m_{0}} E_{P_{S}}\right) \int d \mathbf{q} q_{z}^{2} \mathcal{V}_{q}\left|\int d \mathbf{r} \Psi_{X}^{\alpha} \exp (i \mathbf{q} . \mathbf{r})\right|^{2}
\end{array}\right.
$$

in which $E_{P_{S}}=\left(2 m_{0} / \hbar^{2}\right) P_{S}^{2}$. 
[1] A. Swarnkar, V. K. Ravi, and A. Nag, Beyond Colloidal Cesium Lead Halide Perovskite Nanocrystals: Analogous Metal Halides and Doping, ACS Energy Letters 2, 1089 (2017).

[2] X. Li, Y. Wu, S. Zhang, B. Cai, Y. Gu, J. Song, and H. Zeng, CsPbX3 Quantum Dots for Lighting and Displays: Room-Temperature Synthesis, Photoluminescence Superiorities, Underlying Origins and White Light-Emitting Diodes, Adv. Funct. Mater. 26, 2435 (2016).

[3] S. Ten Brinck and I. Infante, Surface Termination, Morphology, and Bright Photoluminescence of Cesium Lead Halide Perovskite Nanocrystals, ACS Energy Letters 1, 1266 (2016).

[4] L. Protesescu, S. Yakunin, M. I. Bodnarchuk, F. Krieg, R. Caputo, C. H. Hendon, R. X. Yang, A. Walsh, and M. V. Kovalenko, Nanocrystals of Cesium Lead Halide Perovskites $\left(\mathrm{CsPb}_{3}\right.$, $\mathrm{X}=\mathrm{Cl}, \mathrm{Br}$, and I): Novel Optoelectronic Materials Showing Bright Emission with Wide Color Gamut, Nano. Lett. 15, 3692 (2015).

[5] S. Yakunin, L. Protesescu, F. Krieg, M. I. Bodnarchuk, G. Nedelcu, M. Humer, G. De Luca, M. Fiebig, W. Heiss, and M. V. Kovalenko, Low-threshold amplified spontaneous emission and lasing from colloidal nanocrystals of caesium lead halide perovskites, Nat. Commun. 6, 8056 (2015).

[6] Y. Wang, X. Li, J. Song, L. Xiao, H. Zeng, and H. Sun, All-Inorganic Colloidal Perovskite Quantum Dots: A New Class of Lasing Materials with Favorable Characteristics, Adv. Mater. 27, 7101 (2015).

[7] Y. Wang, X. Li, V. Nalla, H. Zeng, and H. Sun, Solution-Processed Low Threshold Vertical Cavity Surface Emitting Lasers from All-Inorganic Perovskite Nanocrystals, Adv. Funct. Mater. 27, 1605088 (2017).

[8] G. Li, Z-K. Tan, D. Di, M. L. Lai, L. Jiang, J- H. Lim, R. H. Friend, and N. C. Greenham, Efficient Light-Emitting Diodes Based on Nanocrystalline Perovskite in a Dielectric Polymer Matrix, Nano Lett. 15, 2640 (2015).

[9] G. Rainò, G. Nedelcu, L. Protesescu, M. I. Bodnarchuk, M. V. Kovalenko, R. F. Mahrt, and T. Stöferle, Single Cesium Lead Halide Perovskite Nanocrystals at Low Temperature: Fast Single-Photon Emission, Reduced Blinking, and Exciton Fine Structure, ACS Nano 10, 2485 (2016).

[10] Y-S. Park, S. Guo, N. S. Makarov, and V. I. Klimov, Room Temperature Single-Photon 
Emission from Individual Perovskite Quantum Dots, ACS Nano 10, 10386 (2015).

[11] F. Hu, H. Zhang, C. Sun, C. Yin, B. Lv, C. Zhang, W. W. Yu, X. Wang, Y. Zhang, and M. Xiao, Superior Optical Properties of Perovskite Nanocrystals as Single Photon Emitters, ACS Nano 12, 12410 (2015).

[12] F. Hu, C. Yin, H. Zhang, C. Sun, W. W. Yu, C. Zhang, X. Wang, Y. Zhang, and M. Xiao, Slow Auger Recombination of Charged Excitons in Nonblinking Perovskite Nanocrystals without Spectral Diffusion, Nano Lett. 16, 6425 (2016).

[13] R. M. Stevenson, R. J. Young, P. Atkinson, K. Cooper, D. A. Ritchie, and J. Shields, A semiconductor source of triggered entangled photon pairs, Nature 439, 179 (2006).

[14] C. L. Slater, R. M. Stevenson, I. Farrer, C. A. Nicoll, D. A. Ritchie, and J. Shields, An entangled light-emitting diode, Nature 465, 594 (2010).

[15] A. Dousse, J. Suffczyński, A. Beveratos, O. Krebs, A. Lemaître, I. Sagnes, J. Bloch, P. Voisin, and P. Senellart, Ultrabright source of entangled photon pairs, Nature 466, 217 (2010).

[16] N. Gisin, G. Ribordy, W. Tittel, and H. Zbinden, Quantum cryptography, Rev. Mod. Phys. 74, $145(2002)$.

[17] X. Li, Y. Wu, D. Steel, D. Gammon, T. H. Stievater, D. S. Katzer, D. Park, C. Piermarocchi, and L. J. Sham, An All-Optical Quantum Gate in a Semiconductor Quantum Dot, Science 301, 809 (2003).

[18] M. Fu, P. Tamarat, H. Huang, J. Even, A. L. Rogach, and B. Lounis, Neutral and Charged Exciton Fine Structure in Single Lead Halide Perovskite Nanocrystals Revealed by Magnetooptical Spectroscopy, Nano Lett. 17, 2895 (2017).

[19] J. Ramade, L. M. Andriambariarijaona, V. Steinmetz, N. Goubet, L. Legrand, T. Barisien, F. Bernardot, C. Testelin, E. Lhuillier, A. Bramati, and M. Chamarro, Fine structure of excitons and electron-hole exchange energy in polymorphic $\mathrm{CsPbBr}_{3}$ single nanocrystals, Nanoscale 10, 6393 (2018).

[20] J. Ramade, L. M. Andriambariarijaona, V. Steinmetz, N. Goubet, L. Legrand, T. Barisien, F. Bernardot, C. Testelin, E. Lhuillier, A. Bramati, and M. Chamarro, Exciton-phonon coupling in a $\mathrm{CsPbBr}_{3}$ single nanocrystal, Appl. Phys. Lett. 112, 072104 (2018).

[21] M. A. Becker, R. Vaxenburg, G. Nedelcu, P. C. Sercel, A. Shabaev, M. J. Mehl, J. G. Michopoulos, S. G. Lambrakos, N. Bernstein, J. L. Lyons, T. Stöferle, R. F. Mahrt, M. V. Kovalenko, D. J. Norris, G. Rainò, and A. L. Efros, Bright triplet excitons in caesium lead 
halide perovskites, Nature 553, 189 (2018).

[22] C. Yin, L. Chen, N. Song, Y. Lv, F. Hu, C. Sun, W. W. Yu, C. Zhang, X. Wang, Y. Zhang, and M. Xia, Bright-Exciton Fine-Structure Splittings in Single Perovskite Nanocrystals, Phys. Rev. Lett. 119, 026401 (2017).

[23] K. Heidrich, W. Schäfer, M. Schreiber, J. Söchtig, G. Trendel, J. Treusch, T. Grandke, and H. J. Stolz, Electronic structure, photoemission spectra, and vacuum-ultraviolet optical spectra of $\mathrm{CsPbCl}_{3}$ and $\mathrm{CsPbBr}_{3}$, Phys. Rev. B 24, 5642 (1981).

[24] J. Even, L. Pedesseau, J-M. Jancu, and C. Katan, Importance of Spin-Orbit Coupling in Hybrid Organic/Inorganic Perovskites for Photovoltaic Applications, J. Phys. Chem. Lett. 4, 2999 (2013).

[25] J. Qian, B. Xu, and W. Tian, A comprehensive theoretical study of halide perovskite $\mathrm{ABX}_{3}$, Organic Electronics 37, 61 (2016).

[26] Z. G. Yu, Effective-mass model and magneto-optical properties in hybrid perovskites, Sci. Rep. 6, 28576 (2016).

[27] P. D. J. Calcott, K. J. Nash, L. T. Canham, M. J. Kane, and D. Brumhead, Identification of radiative transitions in highly porous silicon, J. Phys. Cond. Matter 5, L91 (1993).

[28] M. Nirmal, D. J. Norris, M. Kuno, M. G. Bawendi, Al. L. Efros, and M. Rosen, Observation of the "Dark Exciton" in CdSe Quantum Dots, Phys. Rev. Lett. 75, 3728 (1995).

[29] M. Chamarro, C. Gourdon, P. Lavallard, O. Lublinskaya, and A. I. Ekimov, Enhancement of electron-hole exchange interaction in CdSe nanocrystals: A quantum confinement effect, Phys. Rev. B 53, 1336 (1996).

[30] O. I. Micic, H. M. Cheong, H. Fu, A. Zunger, J. R. Sprague, A. Mascarenhas, and A. J. Nozik, Size-Dependent Spectroscopy of InP Quantum Dots, J. Phys. Chem. B 101, 4904 (1997).

[31] U. Banin, J. C. Lee, A. A. Guzelian, A. V. Kadavanich, and A. P. Alivisatos, Exchange interaction in InAs nanocrystal quantum dots, Superlattices Microstruct. 22, 559 (1997).

[32] G. E. Pikus and G. L. Bir, Exchange Interaction In Excitons In Semiconductors, Zh. Eksp. Teor. Fiz. 60, 195 (1971) [Sov. Phys. JETP 33, 108 (1973)].

[33] G. L. Bir and G. E. Pikus, Symmetry and Strain Induced Effects in Semiconductors (Wiley, New York, 1975).

[34] M. M. Denisov and V. P. Makarov, Longitudinal and transverse excitons in semiconductors, Phys. Stat. Sol. (b) 56, 9 (1973). 
[35] T. Takagahara, Effects of dielectric confinement and electron-hole exchange interaction on excitonic states in semiconductor quantum dots, Phys. Rev. B 47, 4569 (1993).

[36] K. Cho, Unified theory of symmetry-breaking effects on excitons in cubic and wurtzite structures, Phys. Rev. B 14, 4463 (1976).

[37] S. V. Gupalov and E. L. Ivchenko, The fine structure of excitonic levels in CdSe nanocrystals, Physics of the Solid State 42, 2030 (2000).

[38] R. Romestain and G. Fishman, Excitonic wave function, correlation energy, exchange energy, and oscillator strength in a cubic quantum dot, Phys. Rev. B 49, 1774 (1994).

[39] Al. L. Efros, M. Rosen, M. Kuno, M. Nirmal, D. J. Norris, and M. Bawendi, Band-edge exciton in quantum dots of semiconductors with a degenerate valence band: Dark and bright exciton states, Phys. Rev. B 54, 4843 (1996).

[40] A. Franceschetti and A. Zunger, Direct Pseudopotential Calculation of Exciton Coulomb and Exchange Energies in Semiconductor Quantum Dots, Phys. Rev. Lett. 78, 915 (1997).

[41] A. Franceschetti, H. Fu, L. W. Wang, and A. Zunger, Many-body pseudopotential theory of excitons in InP and CdSe quantum dots, Phys. Rev. B 60, 1819 (1999).

[42] E. L. Ivchenko, Optical spectroscopy of semiconductor Nanostructures (Alpha Science, Harrow, UK, 2005).

[43] S. V. Goupalov and E. L. Ivchenko, Electron-hole long-range exchange interaction in semiconductor quantum dots, J. Crystal Growth 184/185, 393 (1998).

[44] A. Franceschetti, L. W. Wang, H. Fu, and A. Zunger, Short-range versus long-range electronhole exchange interactions in semiconductor quantum dots, Phys. Rev. B 58, R13367 (1998).

[45] J. W. Luo, G. Bester, and A. Zunger, Long- and short-range electron-hole exchange interaction in different types of quantum dots, New J. of Physics 11, 123024 (2009).

[46] H. Tong and M. W. Wu, Theory of excitons in cubic III-V semiconductor GaAs, InAs and GaN quantum dots: Fine structure and spin relaxation, Phys. Rev. B 83, 235323 (2011).

[47] S. Sharma, N. Weiden, and A. Weiss, Phase Diagrams of Quasibinary Systems of the Type: $\mathrm{ABX}_{3}-\mathrm{A}^{\prime} \mathrm{BX}_{3} ; \mathrm{ABX}_{3}-\mathrm{AB}^{\prime} \mathrm{X}_{3}$, and $\mathrm{ABX}_{3}-\mathrm{ABX}_{3}^{\prime} ; \mathrm{X}=$ Halogen, Z. Physik. Chem. 175, 63 (1992).

[48] S. Hirotsu, J. Harada, M. Iizumi, and K. Gesi, Structural Phase Transitions in $\mathrm{CsPbBr}_{3}$, J. Phys. Soc. Jpn 37, 1393 (1974).

[49] C. C. Stoumpos, C. D. Malliakas, J. A. Peters, Z. Liu, M. Sebastian, J. Im, T. C. Chasapis, 
A. C. Wibowo, D. Y. Chung, A. J. Freeman, B. W. Wessels, and M. G. Kanatzidis, Crystal Growth of the Perovskite Semiconductor $\mathrm{CsPbBr}_{3}$ : A New Material for High-Energy Radiation Detection, Cryst. Growth Des. 13, 2722 (2013).

[50] C. K. Møller, Crystal Structure and Photoconductivity of Cæsium Plumbohalides, Nature (London) 182, 1436 (1958).

[51] Y. Fujii, S. Hoshino, Y. Yamada, and G. Shirane, Neutron-scattering study on phase transitions of $\mathrm{CsPbCl}_{3}$, Phys. Rev. B 9, 4549 (1974).

[52] J. Even, L. Pedesseau, C. Katan, M. Kepenekian, J.-S. Lauret, D. Sapori, and E. Deleporte, Solid-State Physics Perspective on Hybrid Perovskite Semiconductors, J. Phys. Chem. C 119, $10161(2015)$.

[53] J. Even, Pedestrian Guide to Symmetry Properties of the Reference Cubic Structure of 3D All-Inorganic and Hybrid Perovskites, J. Phys. Chem. Lett. 6, 2238 (2015).

[54] M. O. Nestoklon, S. V. Goupalov, R. I. Dzhioev, O. S. Ken, V. L. Korenev, Yu. G. Kusrayev, V. F. Sapega, C. de Weerd, L. Gomez, T. Gregorkiewicz, J. Lin, K. Suenaga, Y. Fujiwara, L. B. Matyushkin, and I. N. Yassievich, Optical orientation and alignment of excitons in ensembles of inorganic perovskite nanocrystals, Phys. Rev. B 97, 235304 (2018).

[55] In the perovskites studied here, due to large spin-orbit coupling $(\Delta>1 \mathrm{eV})$, a good description of the electron and hole is obtained by the four-band k.p model related to $\Gamma_{6}^{-} \mathrm{CB}$ and $\Gamma_{6}^{+}$VB. In this case, the effective masses of electron and hole, $m_{e}$ and $m_{h}$, can be written as $\left(m_{0} / m_{e}\right)=1+E_{P_{s}} / 3 E_{g}$ and $\left(m_{0} / m_{h}\right)=-1+E_{P_{s}} / 3 E_{g}$. Consequently, $E_{P_{S, \rho}}$ is connected to the reduced mass of exciton, $\mu_{X}$, by the relationship $E_{P_{S, \rho}}=(3 / 2)\left(m_{0} / \mu_{X}\right) E_{g}$. We underline that we have also performed a 40-band k.p calculation leading to $E_{P_{s}}$ values in agreement with the ones estimated from the precedent relationship.

[56] Z. Yang, A. Surrente, K. Galkowski, A. Miyata, O. Portugall, R. J. Sutton, A. A. Haghighirad, H. J. Snaith, D. K. Maude, P. Plochocka, and R. J. Nicholas, Impact of the Halide Cage on the Electronic Properties of Fully Inorganic Cesium Lead Halide Perovskites, ACS Energy Lett. 2, 1621 (2017).

[57] I. Saïdi, R. Ben Aich, M. Yahyaoui, S. Ben Radhia, K. Boujdaria, M. Chamarro, and C. Testelin, Multiband k.p theory of bulk inorganic cesium lead halide perovskites, (to be published).

[58] D. Fröhlich, K. Heidrich, H. Künzel, G. Trendel, and J. Treusch, Cesium-trihalogen-plumbates 
a new class of ionic semiconductors, Journal of Luminescence. 18/19, 385 (1979).

[59] M. Kato, T. Fujiseki, T. Miyadera, T. Sugita, S. Fujimoto, M. Tamakoshi, M. Chikamatsu, and H. Fujiwara, Universal rules for visible-light absorption in hybrid perovskite materials, J. Appl. Phys. 121, 115501 (2017).

[60] S. Makarov, A. Furasova, E. Tiguntseva, A. Hemmetter, A. Berestennikov, A. Pushkarev, A. Zakhidov, and Y. Kivshar, Halide-Perovskite Resonant Nanophotonics, Adv. Optical Mater.2018, 1800784 (2018).

[61] K. Galkowski, A. Mitioglu, A. Miyata, P. Plochocka, O. Portugall, G. E. Eperon, J. T-W. Wang, T. Stergiopoulos, S. D. Stranks, H. J. Snaith and R. J. Nicholas, Determination of the exciton binding energy and effective masses for methtlamnonium and formamidinium lead tri-halide perovskite semiconductors, Energy Environ. Sci. 9, 962, (2016).

[62] M. Sendner, P. K. Nayak, D. A. Egger, S. Beck, Christian Müller, B. Epding, W. Kowalsky, L. Kronik, H. J. Snaith, Annemarie Puccia, and R. Lovrinčić, Optical phonons in methylammonium lead halide perovskites and implications for charge transport, Mater. Horiz. 3, 613 (2016). 